\title{
Das diretrizes à prática: avaliação da atenção pediátrica prestada por um serviço de referência secundária no norte de Minas Gerais
}

${ }^{1}$ Secretaria de Estado de Saúde de Minas Gerais, Governo do Estado de Minas Gerais. Rod. Prefeito Américo Gianetti s/n, Serra Verde. 31630-900 Belo Horizonte MG.

laurinhamcm@yahoo.com.br ${ }^{2}$ Departamento de Pediatria, Faculdade de Medicina, Universidade Federal de Minas Gerais.

${ }^{3}$ Departamento de Medicina Preventiva e Social, Faculdade de Medicina, Universidade Federal de Minas Gerais.
Abstract In the State of Minas Gerais, the Secondary Reference Viva Vida Centers (CVVRS) are one of the strategies deployed to tackle the problems in child health. This study sought to evaluate pediatric care provided in a CVVRS, using the guidelines defined when it was set up as a benchmark. A quantitative-qualitative approach was adopted, which included a cross-sectional study with stratified random sampling of 385 medical records of children registered with the program between 2007 and 2009, and analysis of focus groups with strategic actors of the initiative. There were divergences between the user profiles and the target audience in terms of age, hometown and clinical characteristics. Access and use of the service differed depending on the town, due to problems of misinformation concerning the proposal, difficulty of transportation and the fragility of the health network. The centers are considered an innovative and important initiative for the expansion and organization of the health network, though the intended logic is not effectively seen in practice. Interventions for articulation between the network services and adaptation of the agreed guidelines to the regional specificities are necessary.

Key words Public health policies, Health program and project evaluation, Child health, Secondary healthcare, Primary healthcare
Resumo A coexistência de condições agudas e crônicas demanda a reorganização do sistema de saúde vigente. O Programa Viva Vida é a estratégia de Minas Gerais para enfrentamento dos problemas na área da saúde da criança. Uma de suas ações é a implantação de Centros Viva Vida de Referência Secundária (CVVRS). O objetivo do estudo foi avaliar a atenção pediátrica prestada por um CVVRS, tendo como referência as diretrizes pactuadas para sua implantação. Foi adotada abordagem quantiqualitativa, incluindo estudo transversal com amostra aleatória e estratificada de 385 prontuários de crianças inscritas de 2007 a 2009, e análise de grupos focais com atores estratégicos da iniciativa. Houve divergência entre o perfil dos usuários e o público-alvo previsto em relação à idade, município de origem e características clínicas. $O$ acesso e o uso do serviço são heterogêneos entre os municípios da região devido a entraves como desconhecimento da proposta, dificuldade de transporte $e$ fragilidades da rede. A implantação do centro é identificada como iniciativa inovadora e importante para expansão e organização da rede, entretanto, a lógica planejada não se verifica na prática. Intervenções voltadas para a articulação entre os serviços da rede e a adequação das diretrizes pactuadas às peculiaridades regionais são necessárias.

Palavras-chave Políticas públicas de saúde, Avaliação de programas e projetos de saúde, Saúde da criança, Atenção Secundária à Saúde, Atenção Primária à Saúde 


\section{Introdução}

A atual situação de saúde do Brasil caracteriza-se como uma transição epidemiológica, marcada pela passagem do predomínio de condições agudas para um cenário de expansão de condições crônicas, permanência de doenças infecciosas e parasitárias, além do aumento de mortes por causas externas ${ }^{1}$.

Essa situação torna necessária a revisão do sistema de atenção à saúde vigente de maneira a adaptá-lo a esta realidade. Uma alternativa está na reorganização do sistema em redes de atenção à saúde, estruturadas de maneira poliárquica, com responsabilidades sanitárias e econômicas bem definidas, tendo a atenção primária como centro coordenador, visando à prestação de serviços de saúde no tempo certo, no lugar certo, com a qualidade e custos certos ${ }^{2}$.

Adequando-se a esse modelo de atenção, a Secretaria de Estado de Saúde de Minas Gerais (SES-MG) optou pela implantação de redes prioritárias com base nas situações-problema identificadas no estado. A expressiva presença de causas evitáveis de mortalidade infantil levou à criação da Rede Viva Vida ${ }^{3}$. Para estruturação dessa rede, o Programa Viva Vida vem desenvolvendo, desde 2003, várias ações nos diversos níveis de atenção. Dentre elas, destaca-se a implantação dos Centros Viva Vida de Referência Secundária (CVVRS) ${ }^{4}$.

Esses Centros caracterizam-se como pontos de atenção microrregionais que, atuando de maneira integrada à atenção primária e terciária, devem garantir atenção integral à saúde sexual e reprodutiva e à saúde da criança em situação de risco. Para isso, devem ofertar ações de promoção, prevenção, diagnóstico precoce e recuperação de doenças ${ }^{4}$.

A proposta de implantação dos CVVRS é considerada inovadora uma vez que, além de expandir a oferta na atenção secundária, contribuindo para organização da rede de atenção à saúde da mulher e da criança em Minas Gerais, rompe com a lógica de pagamento por procedimento vigente no SUS, atrelando o financiamento desse ponto de atenção à contratualização de serviços planejados conforme as necessidades de saúde ${ }^{5}$.

Este estudo tem por objetivo avaliar a atenção pediátrica prestada por um CVVRS, tendo como referência as diretrizes pactuadas para sua implantação e a percepção de atores estratégicos sobre o papel e a inserção desse serviço na Rede Viva Vida.

\section{Métodos}

Trata-se de um estudo transversal com abordagem quanti-qualitativa. O local selecionado foi um Centro Viva Vida de uma microrregião de saúde do norte de Minas Gerais, visto que este foi o primeiro CVVRS implantado no estado e é referência para uma microrregião de saúde composta por 16 municípios com características demográficas, econômicas e sociais heterogêneas.

A análise quantitativa contou com uma amostra aleatória e estratificada, calculada com base nas 3.764 crianças inscritas no CVVRS de 2007 a 2009, considerando "prevalência" de 50\% (a que estatisticamente garante a maior amostra necessária), margem de erro de 5\%, intervalo de confiança de $95 \%$, acrescidos de $10 \%$ de perdas, totalizando 385 prontuários a serem estudados.

Para estratificação dos prontuários por município, foi calculada a proporção de menores de cinco anos de cada município em relação ao total de crianças nesta faixa etária na microrregião. Os prontuários foram numerados e sorteados a partir de uma tábua de randomização gerada no pacote estatístico Epi Info 6.046 . Dos 16 municípios da microrregião, dois não constaram na amostra porque não haviam encaminhado pacientes para o CVVRS no período estudado, e quatro apresentaram número de inscritos inferior ao estimado no cálculo da amostra. Essa diferença foi redistribuída pelos demais municípios, observando os mesmos critérios, a fim de manter o total de crianças calculado.

Os dados foram armazenados em formato eletrônico, sem qualquer identificação dos participantes. Para montagem, processamento e análise dos dados foi utilizado o pacote estatístico Epi Info $6.04^{6}$. Foi realizada análise descritiva da distribuição de frequência das variáveis categóricas e análise das medidas de tendência central e de dispersão para as variáveis contínuas. $\mathrm{O}$ teste Qui-quadrado foi utilizado para verificar a associação entre o número de consultas por paciente e as variáveis relacionadas ao perfil dos usuários. Para verificar a associação entre o número de consultas por paciente e as variáveis relacionadas às características do município de origem, foi utilizado o teste de Kruskal-Wallis. O nível de significância adotado foi de $5 \%$.

Além disso, foram realizados grupos focais com atores estratégicos organizados em três grupos. O primeiro foi realizado com profissionais da atenção primária à saúde dos municípios da microrregião (APS) com total de nove participantes $\mathrm{O}$ segundo foi direcionado aos Secretári- 
os Municipais de Saúde da microrregião (GEST). Dos 16 municípios convidados, nove participaram desse grupo. O último grupo foi realizado com a equipe de saúde da criança do CVV, contando com a participação de cinco dos sete profissionais da equipe multidisciplinar (REF).

Os grupos focais ocorreram no dia 6 de abril de 2010, sendo realizada uma reunião com cada grupo, com duração média de 50 minutos. Um roteiro semiestruturado incluindo resultados da análise quantitativa foi utilizado na discussão com os três grupos. Todo o material foi gravado e transcrito. Após a leitura exaustiva do material, a análise foi realizada considerando as seguintes categorias: conhecimento sobre a proposta do CVVRS, utilização do CVVRS, relação entre o CVVRS e a APS, relação entre as diretrizes contratadas e a realidade do serviço.

Este projeto foi aprovado no Comitê de Ética em Pesquisa da UFMG. Todos os participantes foram esclarecidos sobre os objetivos e métodos da pesquisa e assinaram o Termo de Consentimento Livre e Esclarecido.

\section{Resultados e discussão}

Os resultados das análises quanti e qualitativa serão apresentados e discutidos de maneira integrada, organizados em cinco categorias gerais, preservando suas interseções.

\section{Conhecimento sobre a proposta do CVVRS}

O CVVRS é uma das principais estratégias desenvolvidas pela SES-MG para estruturação e organização da Rede Viva Vida, não apenas por expandir a oferta de serviços na atenção secundária, como por ser um ponto de atenção idealizado de acordo com as diretrizes do modelo de redes, apresentando características inovadoras, que o diferenciam de um Centro de Especialidades ${ }^{5}$.

Para garantir que essas inovações sejam compreendidas e operacionalizadas, a SES-MG realizou na inauguração do Centro Viva Vida uma oficina com a participação dos envolvidos com essa iniciativa na microrregião. Entretanto, o que se observa é que o conhecimento sobre a proposta do CVVRS não é uniforme entre os grupos.

Os gestores de saúde e os profissionais da equipe de saúde da criança do CVVRS demonstraram maior clareza sobre os objetivos e público-alvo desse ponto de atenção. Já os profissionais da atenção primária à saúde afirmaram saber pouco sobre a proposta, com exceção de dois que haviam participado do processo de implantação do Centro. Para esse grupo, os gestores e a equipe do CVVRS não explicitaram aos profissionais da APS o papel e as diretrizes de utilização do serviço. A troca de gestão e a alta rotatividade de profissionais foram identificadas como agravantes do problema:

Eu também sei super pouco sobre o CVVRS [...] a gente tem 2 pediatras, então os casos são quase todos resolvidos por lá mesmo [...]. Recentemente que a gente descobriu quantas vagas que a gente tinha aqui [...] as trocas, mudou a gestão [...], acabou que a gente se perdeu um pouco nesse meio $[\ldots]-($ APS $)$

Na proposta do modelo de redes de atenção, a APS é o centro de comunicação da rede, devendo funcionar como elemento coordenador de fluxos e contrafluxos de pessoas, produtos e informações ${ }^{2}$. Esse nível de atenção é considerado o responsável pela classificação de risco e encaminhamento de pacientes para o CVVRS, o que torna imperativa a correção da assimetria de informações observada.

Uma alternativa para a solução desse problema seria a implantação de um sistema de governança capaz de assegurar a institucionalização e o fortalecimento de arenas de discussão - tais como as Comissões Intergestores Bipartite (CIB) - garantindo a responsabilização compartilhada entre estado e municípios ${ }^{7}$.

Os gestores municipais e a equipe do CVVRS também devem assumir uma postura proativa, incentivando a divulgação e o debate sobre as diretrizes e a realidade do serviço. Uma estratégia para superar a distância entre os atores e facilitar a troca de informações, é a criação de Comitês Gestores compostos por gerentes das secretarias municipais de saúde e dos serviços dos diversos níveis assistenciais ${ }^{8}$.

Observa-se ainda que o papel da SES-MG não deve se limitar à elaboração de políticas e realização de ações pontuais. As Gerências Regionais de Saúde e demais estruturas descentralizadas devem acompanhar as mudanças em nível local e promover a articulação e a disseminação da informação entre os vários atores.

\section{Utilização do CVVRS}

A Resolução SES-MG nº. 759/054 define que os Centros Viva Vida de Referência Secundária terão abrangência microrregional, devendo estar localizados no município polo da microrregião, visando garantir o atendimento de toda a população referenciada de acordo com a pactu- 
ação assistencial. O Termo de Compromissos e Metas $^{9}$ firmado entre a SES-MG e o município gestor do CVVRS formaliza essa diretriz, estabelecendo-a como uma obrigação.

Entretanto, a existência desse aparato legal é insuficiente para garantir que a utilização do CVVRS aconteça de forma adequada e homogênea na microrregião. Os resultados encontrados apontam que a utilização do Centro Viva Vida está aquém das metas pactuadas, e que seu uso não se dá de maneira uniforme entre os municípios.

Dos 16 municípios de abrangência do CVVRS em estudo, dois não haviam encaminhado crianças para o Centro no período de análise e outros quatro encaminharam em proporção menor do que a esperada. Do total de crianças inscritas nos dois primeiros anos de funcionamento do serviço, quase $80 \%(\mathrm{n}=2.974)$ eram do município sede. Dentre aquelas que tiveram retorno ao CVVRS, novamente o maior percentual era de pacientes desse município.

A Tabela 1 analisa a relação entre o número de consultas pediátricas no CVVRS e as características do município de origem do paciente. Apenas o número de habitantes do município não apresentou associação, com significância estatística, ao número de consultas de pediatria no CVVRS $(p=0,06)$. A proporção de crianças que tiveram mais de uma consulta pediátrica foi maior quando elas residiam em municípios mais próximos da sede do CVVRS, com menor cobertura da Estratégia de Saúde da Família e Taxa de Mortalidade Infantil mais alta.

Tabela 1. Análise das características dos municípios da microrregião em relação ao número de consultas pediátricas realizadas no CVVRS

\begin{tabular}{lcc}
\hline \multicolumn{1}{c}{ Características } & Mediana & $\begin{array}{c}\text { P } \\
\text { (Kruskal-Wallis) }\end{array}$ \\
\hline $\begin{array}{l}\text { População (habitantes) } \\
01 \text { consulta }\end{array}$ & 9.285 & 0,06 \\
$>1$ consulta & 9.285 & \\
Distância $(\mathrm{Km})$ & & \\
01 consulta & 65,0 & $<0,001$ \\
$>1$ consulta & 46,0 & \\
Cobertura ESF (\%) & & \\
01 consulta & 100 & $<0,001$ \\
$>1$ consulta & 78,8 & \\
Taxa de Mortalidade Infantil & & \\
01 consulta & 17,0 & $<0,001$ \\
$>1$ consulta & 24,0 & \\
\hline
\end{tabular}

Esses achados são coerentes com os argumentos de Unglert et al. ${ }^{10}$ que destacam que a oferta de serviços de saúde não depende simplesmente de sua existência. A acessibilidade deve ser considerada por diversos enfoques, como geográfico, cultural, socioeconômico e funcional. Oliveira et al. ${ }^{11}$ acrescentam que o conceito de acesso no campo da saúde pública é complexo e tende a expressar o grau de ajuste entre as necessidades dos usuários e a oferta de serviços, devendo levar em conta os meios de transporte, a distância, o tempo e o custo de deslocamento.

Essas percepções foram reforçadas pelos participantes dos grupos focais quando abordados sobre a utilização do CVVRS. Foram apresentados mais fatores dificultadores do que facilitadores, indicando que não existe total acessibilidade ao Centro Viva Vida, mesmo existindo a oferta de serviços.

Os fatores facilitadores apresentados limitaram-se à: proximidade geográfica, melhoria do acesso por meio do asfaltamento de estradas da região, boa adesão dos pacientes ao serviço e boa relação do município de origem com o município sede do CVVRS. Este último ponto chama a atenção ao sugerir um viés político para utilização do Centro. Assim, grande é a necessidade de monitoramento da utilização do Centro Viva Vida para evitar qualquer forma de privação ou priorização de atendimento.

Em relação aos fatores dificultadores, a existência de barreiras geográficas, a dificuldade de deslocamento de pacientes, a falta de informação por parte da APS e a alta rotatividade de profissionais foram aspectos bastante abordados pelos grupos. Esse cenário aponta mais uma vez a necessidade de articulação dos diversos atores envolvidos com a proposta do CVVRS a fim de solucionar os impasses identificados.

Outro entrave destacado pelos profissionais do Centro e da APS está relacionado ao sistema de apoio da rede. Para eles, os municípios da microrregião têm dificuldades para realização de exames e dispensação de medicamentos não ofertados no CVVRS, o que compromete o retorno ao serviço no prazo esperado e dificulta a continuidade e a efetividade do tratamento proposto. Este problema remete à questão da atenção integral e evidencia que o arranjo institucional para construção de uma rede regionalizada deve combinar a autonomia de entes federados e a cooperação entre eles para que o princípio da integralidade seja garantido ${ }^{12}$.

Questões relacionadas diretamente ao CVVRS também foram citadas. A estratégia de 
apresentação da proposta de implantação do Centro na região foi considerada inadequada para esclarecer todos os aspectos pertinentes à utilização do serviço.

A rotina operacional do Centro foi criticada, principalmente em relação ao fluxo para agendamento e encaminhamento de pacientes para o serviço. Uma alternativa para esse problema seria a informatização do sistema de marcação, o que permitiria otimizar processos, reduzir custos, diminuir a demora para agendamento, dimensionar a fila de espera e viabilizar o monitoramento do absenteísmo às consultas ${ }^{8}$.

Foi destacada também a inadequação da carteira de serviços contratada pela SES/MG em relação às necessidades de saúde da microrregião, sendo este outro dificultador:

[...] um dos itens que o CVVRS oferece com relação à criança, era, [...] atender crianças com a crise asmática, havia uma meta bem grande. A gente fez uma análise e pelo fato do município ser um município quente, que a temperatura, o clima é diferente, a relação da umidade do ar relativamente boa, a gente percebeu que a gente não tem esse problema como outros municípios de clima mais frio têm. - (REF)

Esse problema também foi constatado por Viegas et al. ${ }^{13}$ que, ao aplicarem sua proposta de metodologia para customização de contratos de saúde aos Centros Viva Vida, concluíram que as metas contratadas pela SES-MG não estão adequadas visto que parâmetros assistenciais estaduais não são capazes de contemplar as peculiaridades regionais. Assim, é necessário rever a carteira de serviços e dos quantitativos contratados de modo a readequar a oferta de serviços a real demanda, otimizando a utilização do CVVRS.

\section{Relação entre o CVVRS e a APS}

No modelo de redes de atenção, a APS é considerada um importante pilar, assumindo o papel central no desenho e funcionamento das redes. Seus atributos são primeiro contato, longitudinalidade, integralidade e coordenação ${ }^{14}$. Dessa forma, sem uma organização mínima da APS, os Centros Viva Vida tendem a se firmar como um centro de especialidades médicas, perdendo seu caráter inovador.

Os participantes reconheceram a importância da atenção primária para o bom funcionamento do CVVRS, mas destacaram que existem entraves que dificultam essa relação, principalmente quanto aos recursos humanos e à organização da rede assistencial.
Em relação aos recursos humanos, os gestores identificaram, dentre outras questões, dificuldades por parte da academia em incorporar as mudanças no modelo assistencial aos seus objetivos educacionais:

A gente tá vendo que no modelo de saúde do Brasil [...] houve uma mudança. A gente viu durante muito tempo que havia investimento sempre na alta complexidade, na média complexidade, em equipamentos, em exames, quando na verdade, o problema era não deixar o paciente adoecer. [...] Então hoje a visão do Estado é essa, evitar que o paciente chegue na atenção secundária fazendo um processo mais barato, mais rápido, mais prático lá na ponta. A deixa é pra que a escola comece a formar profissionais, comece a incluir ai nas disciplinas da medicina uma coisa voltada pra saúde da família, da responsabilidade. - (GEST)

Como consequência, os gestores e a equipe do CVVRS destacaram que os profissionais da APS não têm assumido seu papel, eximindo-se da corresponsabilização pelos pacientes e insistindo no encaminhamento para o CVVRS de casos passíveis de solução na APS.

Campos et al. ${ }^{15}$ afirmam que a formação de profissionais é um processo essencial no desenvolvimento e manutenção de um sistema de saúde, entretanto, esse processo em nível universitário enfrenta problemas que impactam negativamente nessa relação. Existe uma defasagem entre ensino e realidade que precisa ser superada. Nesse sentido, políticas recentes do Ministério da Saúde como o Promed, o Pró-Saúde, o PET-Saúde, a Telessaúde e a UnaSUS vêm buscando reduzir esse distanciamento. Entretanto, os efeitos destas políticas somente serão percebidos no médio e longo prazo.

Problemas relacionados ao mercado de trabalho, tais como dificuldade de contratação e alta rotatividade de profissionais foram mencionados pelos três grupos como dificultadores da relação entre APS e CVVRS. Essa questão não se resume a realidade estudada, sendo bastante discutida na literatura nacional e internacional que reconhece a complexidade do problema e recomenda a combinação de estratégias para superá-lo ${ }^{16-19}$.

No caso brasileiro, a municipalização autárquica e a descentralização dos serviços de saúde aumentaram a responsabilidade dos municípios na gestão do SUS, inclusive nas questões afeitas à gestão do trabalho ${ }^{16-18}$. Além disso, como destaca Mendonça et al. ${ }^{18}$, com a implementação da Estratégia de Saúde da Família como política de fortalecimento da APS, a gestão do trabalho em saúde ganhou ainda mais destaque visto que esse 
modelo assistencial implica a ampliação e a fixação do quadro de pessoal em nível municipal.

Entretanto, na prática, o que se observa são dificuldades técnicas e econômicas por parte dos municípios, o que leva a problemas de contratação, capacitação e fixação de profissionais ${ }^{16,17}$. Campos et al. ${ }^{16}$ ressaltam que muitos municípios, em função de suas frágeis vocações econômicas e de sua menor aptidão para o provimento e a fixação de profissionais de saúde, sofrem com problemas como medidas restritivas para contratação de pessoal no setor público, entre elas a Lei de Responsabilidade Fiscal, com a danosa concorrência que eleva os salários ou ainda implica em concessões que comprometem a qualidade da assistência prestada, além da proliferação de contratos precários.

No caso de Minas Gerais, os resultados do $1^{\circ}$ Censo de Recursos Humanos da Atenção Primária $^{20}$ identificam esses problemas no Estado como um todo e mostram que fatores como vínculo contratual, remuneração e tempo de serviço estão diretamente relacionados à alta rotatividade de profissionais. Dessa forma, esses fatores devem ser considerados nas políticas de recursos humanos, que devem conseguir não apenas fixar e capacitar os profissionais de saúde, mas permitir mecanismos para transmissão de conhecimento, de forma a permitir adequada transição de um profissional para o outro, garantindo a continuidade da qualidade da assistência prestada.

No que tange a rede assistencial, os profissionais da APS destacaram que há um distanciamento entre a realidade do CVVRS e da APS. Tal lacuna faz com que os profissionais da atenção secundária muitas vezes não compreendam as dificuldades enfrentadas no nível primário, identificando-o como não resolutivo. Essa percepção está de acordo com os achados de Villela et al. ${ }^{21}$ que, ao pesquisar os desafios da atenção primária, constatou que para os profissionais da APS os problemas relacionados à estrutura e organização da rede assistencial são os principais obstáculos para o cuidado integral, restringindo a resolutividade da atenção primária e suas possibilidades de atender integralmente as demandas que chegam até ela.

Em relação aos processos e fluxos, os gestores se identificaram como "despachantes de médicos", uma vez que os profissionais da APS solicitam o encaminhamento para especialista e cabe aos gestores solucionar esta demanda. Como não existe outra referência, os gestores acabam utilizando o CVVRS como uma solução para este problema, ainda que este ponto de atenção não tenha este objetivo.
A questão da falta de outra porta. [...] Hoje a porta é essa. Aqui que tem pediatra. Quando abrir outra porta... - (GEST)

O último problema identificado pelos grupos decorre da hegemonia ainda vigente do modelo que valoriza o atendimento especializado. Essa valorização se reflete na pressão das mães pelo atendimento por especialista e não pelo generalista disponível na APS. Essa realidade não apenas implica no aumento de encaminhamentos de casos não previstos na carteira de serviços, como gera uma demanda espontânea não programada para atendimento no CVVRS.

Campos et al. ${ }^{15}$ acreditam que esse tipo de comportamento é decorrente da resistência à mudança do modelo assistencial com o qual os usuários estão acostumados. Nesse sentido, é importante criar estratégias para dar visibilidade ao trabalho e valorizar a atuação dos profissionais da $\mathrm{APS}^{8}$, bem como para incentivar que esses profissionais promovam o acolhimento e fortaleçam o vínculo com os usuários, aumentando a credibilidade e a confiança na capacidade resolutiva desse nível de atenção.

\section{Relação entre as diretrizes contratadas e a realidade do serviço}

No modelo de redes, a organização dos pontos de atenção baseia-se na demanda por serviços e não na oferta indiscriminada dos mesmos. Assim, é fundamental definir o público-alvo a ser atendido e identificar suas necessidades por meio de parâmetros clínicos e assistenciais, para, então, definir, quantificar e orçar a carteira de serviços a ser disponibilizada ${ }^{2}$.

O Termo de Compromissos e Metas ${ }^{9}$, firmado entre a SES-MG e os gestores do Centro, define como público-alvo do CVVRS as crianças menores de cinco anos, dos municípios de abrangência da microrregião, que apresentem alguma das situações de risco estabelecidas na LinhaGuia de Atenção à Saúde da Criança ${ }^{22}$ : prematuridade e/ou baixo peso ao nascer, asfixia perinatal, diarreia de evolução desfavorável, desnutrição moderada/grave, dificuldades respiratórias de evolução desfavorável e triagem neonatal positiva para fenilcetonúria, toxoplasmose, hipotireoidismo, doença falciforme e fibrose cística.

Considerando esses critérios, foi observada divergência entre o perfil dos pacientes atendidos no período de estudo e o público-alvo previsto. A idade de admissão dos pacientes no Centro variou de 2 dias a 16 anos (mediana de 18,6 meses). Uma parcela expressiva de pacientes $(23,1 \%)$ 
apresentou idade superior à proposta para admissão no CVVRS.

No que se refere às características clínicas dos pacientes, chama a atenção o elevado percentual de prontuários sem informação e/ou com letra ilegível, o que impossibilitou a identificação do motivo de encaminhamento em $39,5 \%$ dos prontuários analisados e do diagnóstico do paciente na primeira consulta em $27,3 \%$. O percentual de pacientes com características clínicas não previstas para o CVVRS foi considerado alto $(40,2 \%$ para motivo de encaminhamento e 54,3\% para o diagnóstico). Cerca 20\% das crianças tinham pelo menos uma das condições compatíveis com as indicações para o CVVRS, considerando o motivo de encaminhamento e/ou o diagnóstico.

Em relação à assistência prestada, o pactuado compreendia a prestação dos seguintes serviços, respeitando as orientações dos protocolos clínicos específicos: atendimento com equipe multiprofissional (pediatra, enfermeiro, psicólogo, assistente social, nutricionista e fisioterapeuta), realização dos exames de ultrassonografia, espirometria e pico de fluxo e realização de práticas educativas.

Entretanto, o percentual de crianças que compareceram a consultas de retorno com os pediatras foi baixo $(43,9 \%)$, considerando a proposta de acompanhamento de crianças com condições de risco. Apenas $0,8 \%$ das crianças $(n=3)$ tiveram consultas com nutricionista, o mesmo percentual recebeu atendimentos com a assistente social e somente uma criança $(0,3 \%)$ realizou consultas com a psicologia. A equipe não contava com fisioterapeuta no período estudado. Não há registro de práticas educativas. Em relação aos exames, a espirometria e o pico de fluxo não eram ofertados e a ultrassonografia representou pouco mais de $5 \%$ dos exames solicitados.

Quanto aos dados da primeira consulta, extraídos dos prontuários com informações disponíveis (233 deles para o motivo de encaminhamento; 280 para o diagnóstico; 284 para condutas e 109 para exames), observa-se que apenas $8,14 \%$ de todos os motivos de encaminhamento registrados foram compatíveis com as indicações previstas para o CVVRS. Esse percentual é menor em relação ao diagnóstico (5,45\%). Em relação às condutas, percebe-se que as mais recorrentes foram prescrição de medicamentos $(35,8 \%)$ e pedidos de exames $(30,0 \%)$. O percentual de solicitação de retorno foi considerado baixo, apenas $7,4 \%$.

A Tabela 2 analisa a associação entre características dos pacientes e o número de consultas pediátricas realizadas no CVVRS. Observa-se que houve associação com significância estatística entre o número de consultas pediátricas e o município de origem e a faixa etária do paciente, o que sugere que a proporção de crianças com menos de um mês de idade e residentes no município sede do CVVRS que fizeram mais de uma consulta pediátrica foi maior do que as demais.

Essas divergências entre as diretrizes contratadas e a realidade do serviço foram confirmadas pelos grupos. Os profissionais do CVVRS afirmaram que:

A gente não atende só as crianças indicadas. Atende a população de modo geral. - (REF)

As explicações para essas divergências decorrem de questões já abordadas nas demais categorias desse estudo, tais como:

Tabela 2. Distribuição dos pacientes segundo município de origem, sexo, faixa etária e número de consultas pediátricas no CVVRS

\begin{tabular}{|c|c|c|c|c|c|}
\hline \multirow{2}{*}{ Características } & \multicolumn{2}{|c|}{01 consulta $(n=216)$} & \multicolumn{2}{|c|}{$>1$ consulta $(n=169)$} & \multirow{2}{*}{$\begin{array}{c}\text { P } \\
(\text { Qui-Quadrado) }\end{array}$} \\
\hline & $\mathbf{N}$ & $\%$ & $\mathbf{N}$ & $\%$ & \\
\hline \multicolumn{6}{|c|}{ Município de origem do paciente } \\
\hline Sede & 33 & 34,37 & 63 & 65,62 & $<0,001$ \\
\hline Demais municípios & 183 & 63,32 & 106 & 36,68 & \\
\hline \multicolumn{6}{|l|}{ Sexo } \\
\hline Feminino & 110 & 56,70 & 84 & 43,30 & 0,82 \\
\hline Masculino & 106 & 55,50 & 85 & 44,50 & \\
\hline \multicolumn{6}{|c|}{ Faixa etária do paciente (em meses) } \\
\hline$<1$ & 15 & 42,86 & 20 & 57,14 & 0,04 \\
\hline$>1 \mathrm{e}<12$ & 69 & 53,91 & 59 & 46,09 & \\
\hline$>12 \mathrm{e}<60$ & 87 & 65,41 & 46 & 34,59 & \\
\hline$>60$ & 45 & 50,56 & 44 & 49,44 & \\
\hline
\end{tabular}


- falta de informação dos profissionais da APS, o que compromete o encaminhamento de crianças conforme os critérios estabelecidos;

. fragilidades da rede da atenção, que não é estruturada de maneira uniforme na região, levando ao uso heterogêneo do serviço por parte dos municípios;

- problemas relacionados aos fluxos do CVVRS, dificultando a marcação e o retorno dos pacientes;

. existência de barreiras geográficas e problemas de deslocamento de pacientes, dificultando o acesso ao CVVRS para alguns municípios;

. divergências entre as reais demandas locais e as diretrizes estabelecidas para o CVVRS, o que faz com que os critérios do público-alvo deixem de ser observados para ampliar a oferta de serviços;

. questões culturais relacionadas à dificuldade dos usuários compreenderem a lógica do SUS, pressionando pela garantia de acesso independente dos objetivos estabelecidos para o CVVRS.

No que se refere à atenção prestada no CVVRS, diferente do observado na discussão sobre o público-alvo, não houve consenso entre os grupos em relação a alguns aspectos. Além disso, novos motivos foram apresentados para explicar as diferenças encontradas.

Os grupos apresentaram percepções diferentes sobre o baixo número de consultas por criança no CVVRS no período de estudo. Os gestores e os profissionais da APS afirmaram que esse problema é consequência das diversas dificuldades de retorno ao serviço. Já a equipe do CVVRS defendeu que, apesar de algumas dificuldades, as crianças de fato retornam e têm um acompanhamento no Centro Viva Vida.

Apesar das opiniões discordantes, os grupos defenderam que o retorno aquém do esperado, além de decorrer dos diversos problemas já mencionados, está relacionado ao fato de boa parte dos pacientes encaminhados para o CVVRS não se enquadrar no seu público-alvo. Assim, são acolhidos pela equipe, mas encaminhados para outro serviço mais adequado.

A baixa utilização da equipe multiprofissional do CVVRS também foi relacionada à questão do perfil dos usuários. Para os participantes, o fato de muitas crianças encaminhadas para o CVVRS não serem de risco e, portanto, não precisarem desse tipo de atendimento, não gerou a demanda esperada para a equipe multiprofissional. Além disso, a disponibilidade de alguns desses profissionais no próprio município de origem do paciente reduz ainda mais essa demanda no CVVRS.
Já em relação à realização de práticas educativas e à utilização do plano de cuidado, os grupos foram taxativos quanto à inobservância dessas diretrizes. Os profissionais do CVVRS afirmaram que há a intenção de implantá-las, mas ainda existem dificuldades para organizar a equipe para que o trabalho não se restrinja à realização de consultas.

Os grupos definiram o CVVRS como importante facilitador no processo de encaminhamento para outras especialidades. Contudo, referiram que seu papel é limitado, já que é o município de origem do paciente é o responsável pelo encaminhamento para o especialista e o CVVRS não tem autonomia para gerenciar e utilizar as cotas de atendimento especializado desses municípios. Foi relatado também que há especialidades pouco disponíveis ou indisponíveis na região, o que dificulta ainda mais o encaminhamento dos pacientes para tais serviços.

Os participantes concordaram que o fluxo de referência e contrarreferência ainda não foi implantado na região, entretanto, divergiram em relação aos motivos para esse problema. A equipe do CVVRS afirmou não ter êxito na tentativa de implantação do fluxo devido às fragilidades na APS. Por sua vez, os profissionais da APS atribuíram à equipe do CVVRS a responsabilidade pela não implantação do fluxo, afirmando que referência existe e o que falta é contrarreferência por parte do CVVRS.

Por fim, os gestores e profissionais da APS, apesar das várias críticas, consideraram o serviço resolutivo, atendendo as demandas encaminhadas e contribuindo para a expansão e a organização da rede de atenção à saúde da criança. A equipe do CVVRS também referiu que esse serviço é um ganho para a região, entretanto, destacou que as fragilidades da rede de saúde da região dificultam a capacidade resolutiva do Centro.

\section{Possíveis contribuições \\ para a política da saúde}

Todos os achados reforçam a necessidade de intervenções para que a proposta do CVVRS alcance os resultados esperados. Além da operacionalização das propostas já apresentadas, é fundamental uma reflexão sobre a atual do CVVRS, a fim de adequá-la às demandas e possibilidades da microrregião.

Em primeiro lugar, é preciso repensar as diretrizes estabelecidas para a implantação desse novo serviço considerando as peculiaridades regionais. A proposta do CVVRS dá um impor- 
tante passo ao adotar parâmetros clínicos difundidos na literatura para o estabelecimento da carteira de serviços e definição de metas. No entanto, o uso de parâmetros estaduais não é capaz de refletir as características regionais. Nesse sentido, o uso de indicadores epidemiológicos microrregionais e a construção de uma série histórica de atendimentos de cada CVVRS são importantes estratégias para refinamento da carteira de serviços e das metas a serem contratadas ${ }^{13}$.

A adequação da carteira de serviços também está relacionada a outro importante aspecto da política: o financiamento do CVVRS. Uma vez que custeio desses Centros é calculado com base na estimativa de custo da carteira de serviços, a inadequação desta à realidade local provoca erros no dimensionamento financeiro da proposta, podendo sub ou superestimar os recursos a serem repassados ${ }^{23}$.

Outro foco de discussão deve se concentrar no delineamento de intervenções para reorganização da Rede Viva Vida. Uma vez que o CVVRS foi concebido para operar no modelo assistencial de redes de atenção, seu bom funcionamento depende da estruturação e articulação dos demais elementos que a constituem ${ }^{2}$. Os resultados deste estudo evidenciaram, além de inúmeras dificuldades na atenção primária, fragilidades no sistema de apoio, com a dificuldade de realização de exames complementares e dispensação de medicamentos; no sistema logístico, com problemas no transporte de pacientes e na regulação dos serviços; na atenção especializada, com longas filas de espera para consultas com especialistas. Essa situação demonstra que investimentos isolados não geram resultados esperados, exigindo esforços e incentivos para consolidação de uma rede efetiva.

Outro ponto importante é a necessidade de articulação entre os atores envolvidos com a proposta do CVVRS. Em um contexto federalista, como o brasileiro, a cooperação e a responsabilidade solidária entre as esferas de governo são pontos cruciais para o sucesso das políticas públicas, especialmente na área da saúde ${ }^{12}$. Os cidadãos também têm papel importante nesse processo. É preciso compreender o SUS para melhor utilizá-lo e, principalmente, para poder exercer o controle social, garantindo ganhos permanentes.

Apesar da necessidade dos ajustes citados anteriormente, é possível concluir que a proposta de implantação do CVVRS tem muito a contribuir para expansão e organização da rede de atenção à saúde da criança em Minas Gerais.
É importante reforçar ainda a necessidade de se avaliar políticas públicas, principalmente na área da saúde. Não é possível melhorar sem avaliar. Toda realidade de intervenção sofre influência de diversos fatores o que dificilmente permite que uma política tal como planejada se verifique na prática. Ainda que isso fosse possível, os problemas sociais abordados por políticas públicas, em sua maioria, são complexos, multicausais e passam por transformações ao longo do tempo. Assim, a avaliação tem o papel fundamental de verificar se as ações planejadas e desenvolvidas por determinada política ou programa são válidas, identificar os resultados alcançados, bem como suas fragilidades e potencialidades.

Estudos subsequentes são necessários para cobrir as lacunas deixadas por esta pesquisa. É preciso expandir a avaliação para os demais CVVRS implantados a fim de compreender a realidade de cada microrregião e verificar quais problemas identificados se restringem à realidade do CVVRS estudado e quais são comuns aos outros Centros, permitindo o delineamento mais preciso de macro estratégias. A construção de novos métodos para avaliar a atenção pediátrica superando as limitações deste estudo também é importante, assim como pesquisas para compreender a percepção dos usuários sobre o CVVRS e identificar iniciativas de controle social.

\section{Colaboradores}

LMC Moreira participou da concepção e delineamento do estudo, análise e interpretação dos dados, redação do artigo e aprovação da versão final. CRL Alves e SA Belisário participaram da concepção e delineamento do estudo, interpretação da análise de dados e revisão crítica e aprovação da versão final. MC Bueno e EF Moraes participaram da análise e interpretação dos dados, e revisão crítica e aprovação da versão final. 
Referências

1. Brasil. Ministério da Saúde(MS). Portaria no 4.279/ GM de 30 de dezembro de 2010. Estabelece diretrizes para a organização da Rede de Atenção à Saúde no âmbito do Sistema Único de Saúde (SUS). Diário Oficial da União 2010; $31 \mathrm{dez}$.

2. Mendes EV. As Redes de Atenção à Saúde. 1a Edição. Belo Horizonte: Escola de Saúde Pública de Minas Gerais; 2009.

3. Minas Gerais. Secretaria de Estado de Saúde. Resolução no 356, de 22 de dezembro de 2003. Dispõe sobre o Programa Viva Vida e estabelece outras providências. Diário Oficial dos Poderes do Estado de Minas Gerais 2003; 22 dez.

4. Minas Gerais. Secretaria de Estado de Saúde. Resolução no 759, de 06 de outubro de 2005. Dispõe sobre os Centros Viva Vida de Referência Secundária no âmbito do Estado de Minas Gerais, a instituição de incentivo, no âmbito do Programa Viva Vida, para implantação dos mesmos e dá outras providências. Diário Oficial dos Poderes do Estado de Minas Gerais 2005; 6 out.

5. Lima H, Romanini MAV, Marques AMF, Oliveira MR, Moreira LMC. Rede Viva Vida de Atenção Integral à Saúde da Mulher e da Criança. In: Marques AJS, Mendes EV, Silva JA, Pestana MVC, organizadores. O Choque de Gestão na Saúde em Minas Gerais. Belo Horizonte: Secretaria de Estado de Saúde de Minas Gerais; 2009. p. 111-128.

6. Epi Info [computer program]. Version 6.04. Atlanta (GA): Center for Disease Control and Prevention 2005.

7. Silva MVCP, Júnior FT, Mendes EV. Bases conceituais e programáticas do Choque de Gestão na saúde em Minas Gerais. In: Marques AJS, Mendes EV, Silva JA, Pestana MVC, organizadores. O Choque de Gestão na Saúde em Minas Gerais. Belo Horizonte: Secretaria de Estado de Saúde de Minas Gerais; 2009. p. 29-44.

8. Giovanella L, Mendonça MHM, Almeida PF, Escorel S, Senna MCM, Fausto MCR, Delgado MM, Andrade CLT, Cunha MS, Martins MIC, Texeira CP. Potencialidades e obstáculos para consolidação da Estratégia de Saúde da Família em grandes centros urbanos. Cien Saude Colet 2010; 34(85):248-264.

9. Minas Gerais. Secretaria de Estado de Saúde de Minas Gerais. Resolução no. 1.150, de 19 de abril de 2007. Regulamenta o incentivo financeiro complementar para custeio dos Centros Viva Vida, e estabelece outras providências. Diário Oficial dos Poderes do Estado de Minas Gerais 2007; 19 abr.

10. Unglert CVS, Rosenburg CP, Junqueira CB. Acesso aos serviços de saúde: uma abordagem de geografia em saúde pública. Rev Saude Publica 1987; 21(5) 439-446.

11. Oliveira EXG, Carvalho MS, Travassos C. Territórios do Sistema Único de Saúde - mapeamento das redes de atenção hospitalar. Cad Saude Publica 2004; 20(2):386-402.

12. Menicucci TMG. O Sistema Único de Saúde, 20 anos: balanço e perspectivas. Cad Saude Publica 2009; 25(7):1620-1625.
13. Viegas APB, Moreira LMC, Marques AMF. Uso de indicadores epidemiológicos regionais na avaliação e monitoramento de contratos públicos de saúde. In: VI Conferencia de La Red de Monitoreo y Evaluación de América Latina y el Caribe; 2010; Ciudad de México.

14. Starfield B, Shi L. Policy Relevant Determinants of Health: An International Perspective. Health Policy 2002; 60(3):201-218.

15. Campos FE, Aguiar RAT, Belisário SA. A formação superior dos profissionais de saúde. In: Giovanella L, Escorel S, Lobato LVC, Noronha JC, Carvalho AI, organizadores. Políticas e Sistemas de Saúde no Brasil. Rio de Janeiro: Editora Fiocruz; 2008. p. 1011-1034.

16. Campos FE, Machado MH, Girardi SN. A fixação de profissionais de saúde em regiões de necessidade. Divulgação em Saúde para o Debate 2009; (44):13-24

17. Silveira DS, Facchini LA, Siqueira FV, Piccini RX, Tomasi E, Thumé E, Silva SM, Dilélio AS, Maia MFS. Gestão do trabalho, da educação, da informação e comunicação na atenção básica à saúde de municípios das regiões Sul e Nordeste do Brasil. Cad Saude Publica 2010; 26(9):1714-1726.

18. Mendonça MHM, Martins MIC, Giovanella L, Escorel S. Desafios para a gestão do trabalho a partir de experiências exitosas da expansão da Estratégia da Saúde da Família. Cien Saude Colet 2010; 15(5): 2355-2365.

19. Hutchison B, Abelson J, Lavis J. Primary care in Canada: so much innovation, so little change. Health Affairs 2001; 20(3):116-131

20. Barbosa ACQ, Rodrigues JM, organizadores. Pri meiro censo de recursos humanos da atenção primária do Estado de Minas Gerais. Belo Horizonte: Observatório de Recursos Humanos em Saúde, UFMG; 2006. 144 p.

21. Villela WV, Araújo EC, Ribeiro SA, Cuginotti AP, Hayana ET, Brito FC, Ramos LR. Desafios da Atenção Básica em Saúde: a experiência de Vila Mariana, São Paulo, Brasil. Cad Saude Publica 2009; 25(6): 1316-1324.

22. Minas Gerais. Secretaria de Estado de Saúde. Atenção à saúde da criança. Belo Horizonte: Secretaria de Estado de Saúde de Minas Gerais; 2004.

23. Marques AMF, Souza HF, Moreira LMC. O sistema estadual de monitoramento dos Centros Viva Vida e as bases para a implantação dos sistemas de custos na atenção secundária à saúde em Minas Gerais. Rev. Administração Hospitalar e Inovação em Saúde 2010; 5(jul-dez):56-69.

Artigo apresentado em 30/11/2011

Aprovado em 28/04/2012

Versão final apresentada em 14/05/2012 